\title{
Trauma verstehen und behandeln - eine aktuelle Bestandsaufnahme aus verschiedenen Perspektiven
}

\author{
Silke Birgitta Gahleitner
}

Online publiziert: 21. November 2016

(C) Springer-Verlag Wien 2016

Die Erschütterung über die schwerwiegenden Auswirkungen traumatischer Ereignisse und die entsprechende Aufbereitung in den Medien führt nicht nur in der Allgemeinbevölkerung, sondern auch in der Fachdiskussion häufig zu einer Fokussierung auf die Schäden und Verletzungen traumatisierter Menschen. Dies ist bedauerlich, weil dabei häufig übersehen wird, dass Betroffene mit ihrem Leben auch weiterhin zurechtkommen müssen und dass dies vielen unter beachtlichen Leistungen und Anstrengungen auch gelingt (Birck 2001). Richtet man den Fokus der Aufmerksamkeit daher allein auf die Symptomatik, ignoriert man die faszinierende Überlebenskraft betroffener Menschen. Im vorliegenden Band soll daher bewusst aus sehr unterschiedlichen Perspektiven ein Blick auf die facettenreiche Problematik Trauma geworfen werden. Historische und gesellschaftliche Entwicklungen werden dabei ebenso Thema wie aktuelle klinische Fragestellungen zur Konfrontations- oder Stabilisierungsarbeit mit KlientInnen und PatientInnen. Auch Aspekte transgenerationaler Weitergabe und die Vielfalt verschiedener Arbeitskonzepte in Psychotherapie, Beratung und Traumapädagogik werden im vorliegenden Band angesprochen.

Eingeführt wird der Band durch ein lebendiges Interview mit Katharina Purtscher-Penz und Martin Sack. Unter der Überschrift „Stabilisieren oder Konfrontieren? - Aktuelles aus der Diskussion rund um Traumatherapie“ geben die beiden ExpertInnen aus dem ehemaligen und aktuellen Vorstand der Deutschsprachigen Gesellschaft für Psychotraumatologie Informationen aus erster Hand zu einem bereits sehr lange umstrittenen Thema. Über viele Jahre hinweg wurde und wird immer wieder neu diskutiert, ob

\section{S. B. Gahleitner $(\bowtie)$}

Alice Salomon Hochschule Berlin, Berlin, Deutschland sb@gahleitner.net nun die Traumaexposition den Schlüssel zur Veränderung nach traumatischen Erfahrungen bietet oder Stabilisierung das bessere Angebot auf dem Weg zu einer Heilung darstellt. Die Positionierung der beiden ExpertInnen $\mathrm{zu}$ dieser und einer Reihe von anderen Fragen finden Sie im ersten Beitrag des Themenheftes.

Die historische und gesellschaftliche Perspektive wird insbesondere im zweiten Artikel zum Thema „Transgenerationale Weitergabe traumatischer Erfahrungen" aufgegriffen. Luise Reddemann und Silke Birgitta Gahleitner bemühen sich hier auf eine verständliche Art und Weise um eine offene Spurensuche nicht nur der Folgen des Holocaust für Angehörige der jüdischen Kultur und Religion, sondern auch für die Angehörigen der deutschen und österreichischen Mehrheitsgesellschaft. Eine Gleichsetzung zwischen Opfer- und TäterInnenseite, davon grenzen sich die AutorInnen bereits früh ab, soll damit jedoch in keiner Weise impliziert sein. Vielmehr geht es um das Anliegen, ein besseres Verständnis für Spätfolgen und transgenerationale Phänomene traumatischer Belastungen auf einer breiten Ebene $\mathrm{zu}$ ermöglichen und psychotherapeutisch vermitteln zu können.

Eine neue Versorgungsperspektive für Betroffene traumatischer Belastungen macht Birgit Wagner mit ihrem Artikel „Online-Therapie - eine neue Perspektive in der Psychotherapie für Flüchtlinge und Asylbewerber?" auf. Arabische Flüchtlinge zeigen nachvollziehbarerweise eine hohe Prävalenz von psychischen Störungen. Nach wie vor besteht jedoch ein Mangel an ausreichender psychotherapeutischer und psychiatrischer Versorgung in der Muttersprache. Die ortsunabhängige Kommunikationsform internetbasierter Verfahren bietet hier einen Ausweg. Die Autorin gibt Einblick in ein virtuelles Behandlungszentrum „Ilajnafsy“ und die neuen Möglichkeiten und Grenzen eines solchen internetbasierten Therapieansatzes im arabischen Kontext. 
Ein immer wieder bedeutsames Thema in der Traumatherapie und -beratung greift Karin Pinter auf. Nach wie vor wird der professionellen Beziehungsgestaltung die größte Bedeutung im therapeutischen Prozess zugeschrieben. Unter dem Titel „Affektregulation in der Traumabehandlung - eine bindungsorientierte Perspektive“ werden spezifische Behandlungsprinzipien, die sich an den zentralen Erkenntnissen der Bindungstheorie orientieren, für die Traumatherapie abgeleitet und aufbereitet. Insbesondere die Affektregulation in der Arbeit mit traumatisierten Klientinnen wird an Hand von Fallvignetten aus der klinischen Arbeit äußerst anschaulich ausgeführt und gibt damit einen guten Einblick in die Moment-zuMoment-Arbeit mit traumatisierten PatientInnen.

Traumatische Belastungen stellen jedoch nicht nur PsychotherapeutInnen, sondern alle psychosozialen Fachkräfte vor große Herausforderungen. Andererseits bietet eine individuell abgestimmte und adäquate Traumabehandlung große Chancen für die Betroffenen - bis hin zu einem posttraumatischen Wachstum. Auf der Basis eines gemeinsamen Grundverständnisses von Traumaentstehung und -bewältigung entfaltet ein weiterer Artikel daher unter dem Titel „Traumatherapie, Traumaberatung, Traumapädagogik - ein aktueller Überblick über neue Interventionsformen bei komplexen Traumafolgestörungen“ Gemeinsamkeiten und Unterschiede zwischen verschiedenen Behandlungsformen. Die einzelnen Vorgehensweisen werden von Silke Birgitta Gahleitner und Christina Rothdeutsch praxisorientiert anhand eines gemeinsamen Grundmodells veranschaulicht - ein Plädoyer für eine adäquate Hilfeplanung und -umsetzung.

Einige Überlegungen zum Abschluss von Henriette Löffler-Stastka bieten eine gelungene Praxisreflexion unter dem Titel: „Psychiatrie in der Medizin Psychotherapie in der Medizin Zuhören - Verarbeiten - In Beziehung sein - Intervenieren“ zur Rolle der Psychiatrie und Psychotherapie in der Medizin. Um diesem Gegenstandsbereich gerecht $\mathrm{zu}$ werden, gilt es die Subjektivität in den Fokus zu stellen und sich vor allem zur gesellschaftskritischen Aufgabenstellung der Psychoanalyse zu Wort zu melden. Die AG Ambulante Psychotherapie der Österreichischen Gesellschaft für Psychiatrie, Psychosomatik und Psychotherapie (ÖGPP) hat im Rahmen der Jahrestagung $2015 \mathrm{zu}$ einem Workshop und einer Diskussion rund um dieses Thema eingeladen. Die Autorin reflektiert die dortigen Überlegungen in einer anregenden und gelungenen Form.

Wir hoffen, dass diese breite Zusammenstellung der Artikel unsere LeserInnen anregen kann, auf einer ebenso großen Breite Anstöße zu erhalten und neue Ideen zu entwickeln, wie traumatisierten Menschen noch engagierter und besser geholfen werden kann.

Interessenkonflikt S.B. Gahleitner gibt an, dass kein Interessenkonflikt besteht. 